\title{
Analyse de la végétation pâturée autour des ouvrages hydrauliques dans le Département du Batha Est au Tchad
}

\author{
A.B. BECHIR ${ }^{1 *}$ et Y. Mopaté LOGTENE ${ }^{2}$ \\ ${ }^{1}$ Université des Sciences et de Technologie d'Ati, BP 9957, Ati, Tchad. \\ ${ }^{2}$ Institut de Recherche en Elevage pour le Développement (IRED). Ex Laboratoire de Recherches Vétérinaires \\ et Zootechniques, BP 433, N'Djaména, Tchad. \\ *Auteur correspondant ; E-mail : abrabechir@yahoo.fr ; Tél : (+235) $66254261 /(+235) 91003574$
}

\section{RESUME}

Une analyse floristique des pâturages situés à proximité des points d'abreuvement des animaux a été effectuée. L'objectif de l'étude a été d'analyser la végétation pâturée dans le contexte de fortes pressions pastorales provoquées par l'installation de ces ouvrages hydrauliques. Les méthodes utilisées ont été celles de relevés phytosociologiques de Braun-Blanquet. L'étude a permis de connaître la diversité floristique et les potentialités fourragères et productives des parcours pastoraux du Batha Est. L'inventaire floristique a montré une flore riche (158 espèces réparties dans 111 genres et 41 familles) à importance agrostologique très variable et repartie dans six (6) unités pastorales. La forte proportion des poaceae et des légumineuses confère à la zone d'étude un intérêt pastoral certain. Elle a également montré une nette diminution de la richesse spécifique herbacée et ligneuse à proximité des points d'abreuvement, avec une tendance à l'homogénéisation de la végétation. Bien que la charge réelle des parcours apparaisse dépassée, aucun effet lié à la présence d'un point d'eau sur la biomasse herbacée n'a été observée. Afin de sécuriser les systèmes pastoraux dans la zone, toute stratégie doit s'appuyer sur un aménagement du territoire et une gestion concertée de l'espace et des ressources.

(C) 2015 International Formulae Group. All rights reserved.

Mots clés : Végétation, pâturage, hydraulique pastorale, Batha Est, Tchad.

\section{INTRODUCTION}

Le Batha est l'une des principales Région d'élevage du Tchad. A l'instar des autres pays sahéliens, les ressources en eau souterraine y sont importantes; mais, ce sont plutôt les pâturages et leur gestion qui constituent un défi majeur. C'est pourquoi, pour optimiser l'exploitation de ces parcours pendant la saison sèche, des ouvrages hydrauliques y ont été installés ou aménagés. Cette nouvelle dynamique a provoqué des déplacements du cheptel centrés sur les points d'eau dont le nombre ne cesse d'augmenter. L'une des conséquences est le problème d'occupation de l'espace en raison de l'afflux massif et de la forte concentration du cheptel autour de ces ouvrages. La mise en place de la politique d'hydraulique a ainsi provoqué de profonds changements non seulement sur l'économie pastorale de la zone, mais aussi sur la gestion de l'espace et des ressources par les éleveurs. Du point de vue 
environnemental, la fixation des troupeaux autour des points d'eau permanents provoque une dégradation des écosystèmes pastoraux (Bonnet et al., 2005). En effet, les ressources en eau structurent géographiquement l'espace pastoral et les abreuvement rythment les déplacements du bétail (Toutain et al., 2012). Ainsi, selon la densité des ouvrages et leur statut juridique, les différents parcours de la zone ont connu des modifications plus ou moins profondes. Les conséquences sont la diminution des pâturages naturels consécutive à une forte concentration du cheptel et à une augmentation rapide des mises en culture des espaces pastoraux. Plusieurs études sur les pâturages sahéliens et sahariens (Béchir et al., 2004 ; Tarhouni et al., 2007; Béchir et Mopaté, 2015) ont montré une dégradation progressive du couvert végétal à proximité des ouvrages hydrauliques. C'est pourquoi la dégradation de l'environnement par les activités pastorales en rapport avec les points d'abreuvement est devenue une préoccupation pour les projets qui interviennent au Tchad en hydraulique pastorale. C'est pour anticiper sur ce phénomène de dégradation écologique que la présente étude a été menée dans le Batha Est. Elle vise l'analyse de la végétation pâturée de la zone dans un contexte de forte pression foncières et pastorales afin d'établir une situation initiale ou situation de référence " $\mathrm{t}_{0}$ ". De plus, l'étude permettra de tirer des conclusions sur des risques éventuels de dégradation de certains écosystèmes très fragiles et sensibles et d'en déduire les conséquences pour le pastoralisme dans la zone.

\section{MATERIEL ET METHODES}

\section{Présentation de la zone d'étude}

Situé au centre du Tchad, la Région du Batha s'étend entre le $12^{\text {ème }}$ et le $16^{\text {ème }}$ parallèle Nord avec une superficie de 88800 $\mathrm{km}^{2}$ soit $21 \% \mathrm{du}$ territoire national. Elle comprend trois (3) départements administratifs dont le département du Batha
Est qui a servi de cadre à la présente étude (Figure 1).

Le Batha Est est sous l'influence d'un climat de type saharien subdésertique au Nord et semi-aride sahélien au Sud. La saison sèche s'étale d'octobre à mai et la saison des pluies de juin à septembre. Les températures oscillent entre 14 et $42{ }^{\circ} \mathrm{C}$. Le relief de la zone est peu accidenté. La zone est parcourue par des cours d'eaux en majorité temporaires et endoréique, dont la durée des écoulements dépend de la variabilité de la pluviométrie et de leur position dans la région. Les sols sont constitués des formations sableuses appelées encore «Goz» en langue locale caractérisés par la prédominance des sols argilo-limoneux compacts ou «Naga» au Nord. Ce sont de grands ensablements dunaires et des plateaux à dépressions fermées ou «ouadis » parfois très rapprochés.

La plus grande partie du Batha Est est occupée par une végétation des dunes, de basfonds et des glacis constituée des steppes arbustives basses à épineux très clair semées et parfois denses à moyennement denses. C'est une végétation à dominance herbacée sous forme de tapis plus ou moins continu composé de Cenchrus biflorus, Dactyloctenium aegyptium, Schoenefeldia gracilis, Aristida spp. et Eragrostis tremula. La strate ligneuse est composée d'Acacia tortilis, Balanites aegyptiaca, Maerua crassifolia, Leptadenia pyrotechnica, Acacia nilotica et Boscia senegalensis.

\section{Choix des zones de relevés}

Pour répondre aux différents critères de représentativité et d'homogénéité, cinq (5) puits ont fait l'objet d'observations pour un total de dix lignes ou transects, matérialisées dans six différents sites (Figure 2).

- deux lignes orientées Est/Ouest et Nord/Sud [Lignes K et L] ;

- deux lignes orientées Est/Ouest et Nord/Sud [Lignes $\mathrm{M}$ et $\mathrm{N}]$; 
- deux lignes orientées Est/Ouest et Nord/Sud [Lignes $\mathrm{O}$ et $\mathrm{P}]$;

- deux lignes orientées Est/Ouest et Nord/Sud [Lignes Q et R].

- deux lignes orientées Est/Ouest et Nord/Sud [Lignes $\mathrm{S}$ et $\mathrm{T}]$.

Des relevés ont été également effectués dans les intersections et les liaisons entre deux points d'eau consécutifs. La recherche des conditions d'homogénéité floristique et écologique des différentes stations avant l'exécution des relevés a nécessité des ajustements géographiques par rapport aux coordonnées retenues préalablement. Pour garantir les conditions d'homogénéité, les zones de transition floristique ont été évitées.

\section{Méthode d'étude de la végétation}

En raison de la variabilité spatiale des peuplements, nous avons utilisé la méthode d'échantillonnage aléatoire stratifié. La méthode phytosociologique de Braun Blanquet (1932) qui permet de décrire la flore et la structure d'une végétation a été utilisée au cours des relevés. Une liste floristique exhaustive de toutes les espèces a été ensuite dressée et chaque espèce herbacée ou ligneuse s'est vue attribuer une cote d'abondance dominance selon un ratio abondance recouvrement :

- 5 pour l'espèce couvrant 65 à $100 \%$ de la surface du relevé ;

- 4 pour celle qui couvre 35 à $65 \%$ de la surface du relevé ;

- 3 pour l'espèce qui couvre 10 à $35 \%$ soit le 1/3 de la superficie du relevé ;

- 2 quand l'espèce est peu abondante et ne couvre que 5 à $10 \%$ de la surface du relevé ;

- 1 espèce peu abondante mais à recouvrement faible, soit moins de $5 \%$ du relevé ;

- (+) pour l'espèce présente à l'état d'individus rares, couvrant moins de $1 \%$ du relevé.
Cette cote est complétée pour chacune des strates par une double observation: une première sur la végétation strate par strate et une seconde observation sur l'état de la surface du sol. Ces observations ont été faites suivant cinq aspects: la géomorphologie, la nature du sol et la proportion de sol nu d'une part, et l'action du vent ou de l'eau d'autre part. La structure de la végétation a été estimée visuellement tandis que les coordonnées sont repérées au GPS (Global Positioning System). Cette méthode a permis par échantillonnage, de couvrir d'importantes surfaces.

En raison de la charge animale réelle peu connue, l'étude de l'état de la strate herbacée a été basée sur des critères botaniques auxquels ont été associés des critères fourragers (appétibilité, valeur pastorale...) et écologiques (catégories d'espèces, réaction à la pâture et le substrat). L'information recueillie a été d'une précision assez suffisante pour établir la typologie des différentes unités de pâturage et de se rendre compte de leur état à différentes échelles ainsi que de leur potentialité. Les fréquences ont permis d'exprimer l'occurrence des espèces : les espèces fréquentes sont celles qui sont recensées dans au moins $50 \%$ des relevés; les espèces peu fréquentes sont celles qui sont présentes dans 25 à $49 \%$ de relevés et les espèces rares sont celles présentes dans moins de $25 \%$ de relevés.

Les données climatiques de la Région ont été décrites à partir de la station synoptique de la ville d'Oum Hadjer chef-lieu du Département de Batha Est.

\section{Analyse de la productivité et capacité de charge des herbages}

La disponibilité fourragère des parcours a été appréhendée à partir de la répartition de la biomasse végétale produite en rapport avec un point d'eau existant ou un campement. Un pâturage n'est réellement exploitable par les animaux que lorsqu'il se trouve à une distance 
maximum de 15 à $20 \mathrm{~km}$ d'un point d'eau (Thébaud, 1990). Pour cette raison, la quantité moyenne de production végétale a été estimée pour les différentes zones situées en moyenne à des distances minimum de 15 à $20 \mathrm{~km}$.

La biomasse herbacée a été évaluée par la méthode de la récolte intégrale. Des coupes ras ont été effectuées à l'intérieur des placettes de $1 \mathrm{~m}^{2}$ placées de façon aléatoire tous les 20 mètres le long des transects. Le nombre de placettes récoltées et pesées a été de 203. La récolte de chaque placette a été pesée immédiatement à l'aide de pesons à ressort de $500 \pm 5 \mathrm{~g}$ et $1000 \pm 10 \mathrm{~g}$. Afin de déterminer le taux de matière sèche, un échantillon de $500 \mathrm{~g}$ a ensuite été séché à l'étuve à $105^{\circ} \mathrm{C}$ pendant $48 \mathrm{~h}$. La biomasse, égale au poids de matière sèche de l'ensemble des organes aériens vivants des espèces par unité de surface a été exprimée en tonne de matière sèche à l'hectare et par an (tMS/ha/an).

La charge maximale en Unité de Bétail Tropical (UBT) qu'un pâturage ou un point d'eau peut supporter a été calculée à partir de la biomasse végétale disponible et de la consommation moyenne par UBT. Elle a été ensuite comparée avec la charge réelle (exprimée en UBT) qui est constituée par les effectifs d'animaux fréquentant les zones. Le ratio entre la charge effective et les possibilités d'accueil donne une indication assez claire sur les actions à envisager pour éviter des surpâturages.

\section{Le spectre biologique ou formes biologiques}

Pour apprécier la réaction d'un pâturage aux contraintes du milieu, il est important de classer les plantes selon la classification de Raunkiaer (1934). Adaptée par plusieurs auteurs pour l'étude des formations végétales tropicales, cette classification tient compte essentiellement de la position des bourgeons et de la taille de l'individu.

\section{Analyse des données}

L'analyse floristique qualitative (famille, genre, espèces) a été réalisée sur la base de la liste floristique générale des différents relevés. L'indice de diversité de Shannon $(\mathrm{H})$ a été utilisé pour caractériser la diversité floristique des différentes unités de végétations pâturées. Il a été calculé pour chaque unité de végétation et pour l'ensemble de la zone par la formule :

\section{$\mathrm{H}=-\sum[(\operatorname{CSp}(i) / C S p) \times(\operatorname{LnCSp}(i)$ \\ $/ C S p)]$ Où}

$\mathrm{H}$ est l'indice de diversité de Shannon

CSp(i), la contribution spécifique de présence de l'espèce $i$;

CSp, la contribution spécifique de toutes les espèces.

La diversité est faible lorsque $\mathrm{H}$ est inférieur à 3 bits, moyenne si $\mathrm{H}$ est compris entre 3 et 4 bits puis élevé quand $\mathrm{H}$ est supérieur ou égal à 4 bits.

Le coefficient de similitude de Sorensen (1948) a été également utilisé pour évaluer la ressemblance floristique entre les différentes unités de végétation. Il a permis d'apprécier le degré de ressemblance entre les listes d'espèces de deux différents sites :

$$
C s=\frac{2 a}{(2 a+b+c)}
$$

Dans cette formule, a représente le nombre d'espèces du site $\mathrm{A}, \mathbf{b}$ celui du site $\mathrm{B}$ et $\mathbf{c}$ le nombre d'espèces communes aux deux sites A et B.

- L'indice d'équitabilité (E) de Pielou (1969) a été utilisé pour décrire la répartition des effectifs des différentes espèces des peuplements. Il a été calculé par la formule :

\section{$E=H / \log _{2} S$}

Où $\mathrm{H}$ est l'indice de diversité de Shannon; $\log _{2} \mathrm{~S}=\mathrm{H}_{\text {max }}$ où $\mathrm{S}$ est le nombre total d'espèces et $\log _{2} \mathrm{~S}$ est la diversité théorique maximale. $\mathrm{E}$ est compris entre 0 et 1. E tend vers 0 lorsque la quasi-totalité des individus appartiennent à une seule espèce et prend la valeur 1 lorsque toutes les espèces ont exactement le même recouvrement. 


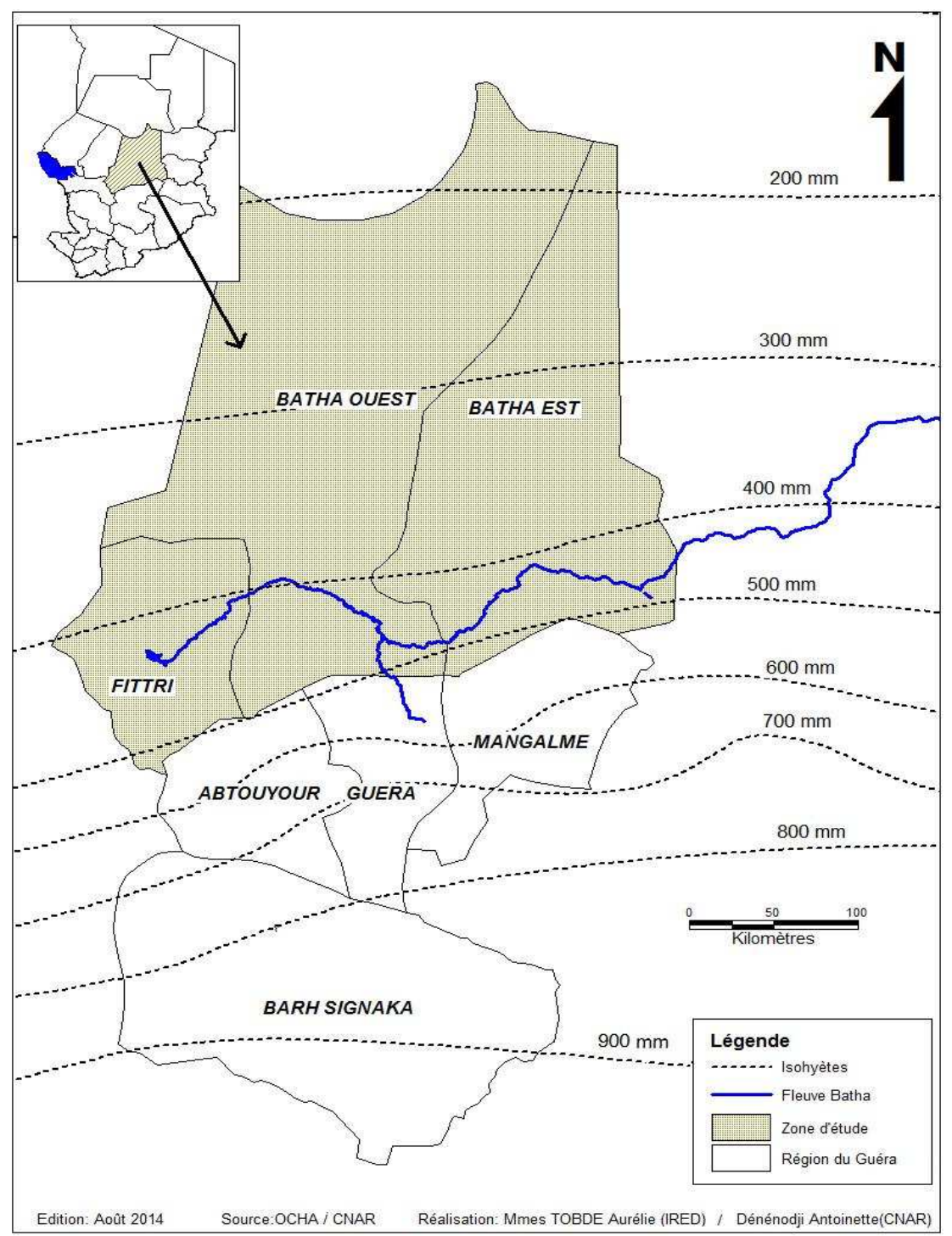

Figure 1 : Localisation de la zone d'étude.

\section{RESULTATS}

\section{Pluviosité}

La moyenne des précipitations sur 20 ans (1990-2013) a été de 373, $70 \mathrm{~mm}$ avec un coefficient de variation de $25 \%$. Le maximum de pluies a été de $673,33 \mathrm{~mm}$. Les années 1990, 2002 et 2009 ont été les plus mauvaises car les cumuls annuels et la hauteur moyenne d'eau tombée ont été faibles. En revanche, les années 1994, 1998, 2008 et 2010 ont été bonnes en raison des cumuls annuels apparemment plus élevés mais aussi d'une meilleure distribution des événements pluvieux (Figure 3).

Richesse et diversité floristiques de la zone Diversité qualitative des différents sites

$\mathrm{Au}$ total, 196 relevés phytosociologiques ont été effectués sur les différents sites retenus et ont concerné les strates ligneuse et herbacée. Cent cinquante huit (158) espèces dont 114 herbacées $(72,15 \%)$ et 44 ligneux $(27,84 \%)$ ont été recensées et réparties en 111 genres appartenant à 41 familles. La famille des 
Poaceae vient en tête par le nombre d'espèces $(18,32 \%)$ suivie par les Mimosaceae $(7,60 \%)$ et les Fabaceae $(5,70 \%)$.

La fréquence spécifique

Le spectre des fréquences des espèces inventoriées indique que seulement $20,15 \%$ des espèces ont été fréquentes sur les différents sites d'étude (Figure 4). En effet, elles ont été recensées dans au moins $50 \%$ des relevés. Il s'agit de Cenchrus biflorus (81,34\%), Acacia tortilis (71,33\%), Balanites aegyptiaca (69,06\%), Schoenefeldia gracilis (64,41\%), Eragrostis tremula (61,32\%), Dactyloctenium aegyptium (60,45\%), Brachiaria deflexa $(58,41 \%)$, Calotropis procera $(54,32 \%)$, Chrozophora senegalensis (50,45\%), Zornia glochidiata $(47,28 \%)$ et Alysicarpus rigosus (42,08\%). Les espèces peu fréquentes ont été de l'ordre de 31,73\%. Certaines espèces ont été cependant rares $(48,12 \%)$. Ce sont: Guiera senegalensis, Maerua crassifolia, Tripogon minimus, Diospyros mespiliformis, Cassia mimosoides, Piliostigma reticulatum, Tamarindus indica, Hyphaene thebaica, et Cordia sinensis (Figure 4).

\section{Spectre biologiques}

L'analyse des types biologiques a montré une prééminence des Thérophytes $(66,13 \%$ de spectre brut et $48,12 \%$ de spectre pondéré) et des Phanérophytes $(21,23 \%$ de spectre brut et $53,12 \%$ de spectre pondéré) sur les autres formes biologiques. Ils sont suivis de très loin par les Hémicryptophytes (6,32\% de spectre brut et $4,51 \%$ de spectre pondéré) et les Chaméphytes $(4,21 \%$ de spectre brut et $1,38 \%$ de spectre pondéré). Les géophytes ont été faiblement représentés (Figure 5).

\section{Les principales grandes unités pastorales identifiées}

Selon la couverture végétale et la diversité floristique, six (6) unités pastorales ont été identifiées.

- Unités pastorales I : formations de basfonds et bordures des cours d'eau et mares : Ce sont des unités de végétation des thalwegs et dépressions fortement représentées dans la zone. La strate ligneuse très dense a été dominée par Mitragyna inermis, Acacia nilotica et Bauhinia rufescens alors que la strate herbacée très variable a été dominée par Echinocloa colona, Panicum laetum et Bergia suffritucasa. En revanche, Oriza barthii, Panicum laetum, Sesbania pachycarpa et Echinochloa colona espèces caractéristiques des dépressions cèdent parfois la place à Bergia suffritucosa, Glinus latioides, ou à des sols complètement dénudés. L'apport en limon a aussi assuré un équilibre instable à d'autres espèces herbacées des milieux hydromorphes comme Sphenoclea zaylanica, Glinus latioides, Ammania auriculata et Bergia sufritucosa. Ces dernières espèces sont de valeur pastorale nulle. Ces types de formations ont également montré une grande richesse floristique ligneuse (10 espèces en moyenne).

- Unités pastorales II : steppes herbeuses à arbustives des ensablements et dunes : Elles occupent de vastes étendues sans variations importantes et présentent une flore riche et variée qui forment un ensemble prairial assez clair, plat avec très peu d'arbres. La couverture végétale a été répartie de façon homogène et a semblé moins affectée qu'ailleurs. La strate ligneuse a été dominée par Acacia tortilis, Balanites aegyptiaca, Ziziphus mauritiana et Acacia mellifera. Les principales espèces herbacées dominants identifiées ont été: Cenchrus biflorus, Schoenefeldia gracilis, Aristida mutabilis et Brachiaria deflexa.

- Unités pastorales III : steppes herbeuses à arbustives des glacis : Ces formations ont occupé le plus souvent les sols sablonneux, pauvres et parsemés de quelques rares épineux. La strate herbacée dominée par Cenchrus biflorus, Dactyloctenium aegyptium, Eragrostis tremula, Aristida mutabilis ou Schoenefeldia gracilis a été très aérée. Selon l'abondance-dominance des différentes espèces, ces types de formations ont été rencontrés dans toute la zone d'étude. Les espèces présentes y ont formé une étendue assez continue de prairies. Les ligneux les plus fréquents ont été : Acacia tortilis, Balanites aegyptiaca, Hyphaene thebaica et Calotropis procera. La strate herbacée a varié localement selon l'importance de Schoenefeldia gracilis, d'Eragrostis tremula 
ou d'Aristida mutabilis qui n'ont pas connu une distribution régulière. Lorsque ces espèces ont été très bien distribuées, il a été notés des faciès à Eragrostis tremula et Aristida mutabilis, de variantes à Schoenefeldia gracilis et Eragrostis tremula et enfin des faciès à Schoenefeldia gracilis et Aristida mutabilis.

- Unités pastorales IV : les formations des plateaux sableux entaillés de dépression fermées ou «Ouadis»: Ces faciès ressemblent beaucoup au précédant par leur composition floristique à tendance agrostologiques marquée. Ils ont été constitués d'espèces fourragères herbacées tendres associées à d'autres dont le développement a été favorisé par l'intervention de l'homme et de l'animal. La strate herbacée a été dominée par Brachiaria deflexa et Cenchrus biflorus. En sous étage, les autres espèces comme Polycarpea corymbosa, Fimbristilis exilis, Bergia suffritucosa, Glinus latioides et Spermacoce radiata ont été toujours présentes mais à l'état diffus. Assez lâche, la strate arbustive a été dominée par Combretum glutinosum, Acacia tortilis et Balanites aegyptiaca. Sur l'ensemble des 40 à $60 \%$ de recouvrement de la végétation herbacée, Brachiaria deflexa a occupé environ $30 \%$.

- Unités pastorales $V$ : les formations des massifs dunaires et des creux interdunaires: les contraintes édaphiques associées aux faibles précipitations dans la zone ont limité le développement de la flore phanérohytes qui a été essentiellement représentée par Acacia tortilis, Balanites aegyptiaca et Leptadenia pyrotechnica. Très hétérogène, la strate herbacée a été dominée par Schoenefeldia gracilis, Cenchrus biflorus, Eragrostis tremula et parfois Chrozophora senegalensis. Elle a été répartie généralement entre les interstices concentriques de plus en plus étroits de l'extérieur vers le centre de la dépression. La densité de la couverture végétale a augmentée dans les inter-dunes avec l'apparition de Maerua crassifolia, arbustes à feuilles crassulescentes. Cette espèce a été absente en haut de pente et sur les crêtes plus ou moins mobiles où ont cependant subsisté quelques espèces reviviscentes comme Aristida spp. ou sarmenteuse telle que Chrozophora senegalensis. La flore arbustive a été ensuite enrichie avec l'apparition de Capparis decidua, Acacia tortilis et Boscia senegalensis toujours accompagnés de Balanites aegyptiaca. Ces ligneux ont été persistants sur les dômes et les pentes dunaires mais ont disparu lorsque l'inter-dune devient trop accentuée.

- Unités pastorales VI: les parcours anthropiques ou formations des jachères : elles ont été caractérisées par une flore très marquée en raison de la forte influence des activités anthropiques. Ce sont des jachères de 2 à 3 ans où l'humidité assurée par la présence des cours d'eau ont permis le développement d'une strate herbacée assez bien fournie. Elle a été dominées parCenchrus biflorus, Schoenefeldia gracilis, Aristida mutabilis, Eragrostis tremula et Loudetia simplex. Les espèces ligneuses répertoriées ont été : Guiera senegalensis, Ziziphus mauritiana, Calotropis procera, Leptadenia pyrotechnica, Combretum glutinosum, Balanites aegyptiaca et Acacia tortilis.

Productivité et capacité de charge des principales unités pastorales identifiées

Bien que constitués majoritairement des espèces annuelles, les pâturages du Batha Est ont montré une productivité comprise entre 1 à 7 tMS/ha/an, avec une charge annuelle théorique variant entre 0,25 et 1,5 UBT/ha (Tableau 1).

Diversité de la flore des différentes unités pastorales

Richesse spécifique, Indice de Shannon et Equitabilité de Pielou

Globalement, toutes les unités pastorales identifiées ont montré un indice d'équitabilité assez élevé (Tableau 2). Au niveau des écosystèmes pâturés, ce sont les unités I et IV qui ont montré les plus grandes valeurs d'indice de diversité de Shannon $(2,96$ $\pm 0,55$ et 2,88 $\pm 0,39$ respectivement). La plus faible richesse floristique a été observée au sein de l'unité V (21 espèces seulement). En revanche, c'est au niveau des unités II et III que les richesses floristiques ont été les plus importantes $(48$ et 43 espèces respectivement). Ces unités ont enregistré les 
plus faibles indices de diversité $(2,52 \pm 0,55$ et 2,73 $\pm 0,32$ Bits respectivement).

\section{Indice de similitude de Sorensen}

Le coefficient global de similitude de Sorensen entre les différentes formations a varié entre 21 et $43 \%$ (Tableau 3). Les unités
II et III ont montré la plus forte similarité (43\%), suivies des unités I et V (37\%). La plus faible similitude a été observée entre les unités I et VI (21\%), suivie des unités I et II $(22 \%)$.

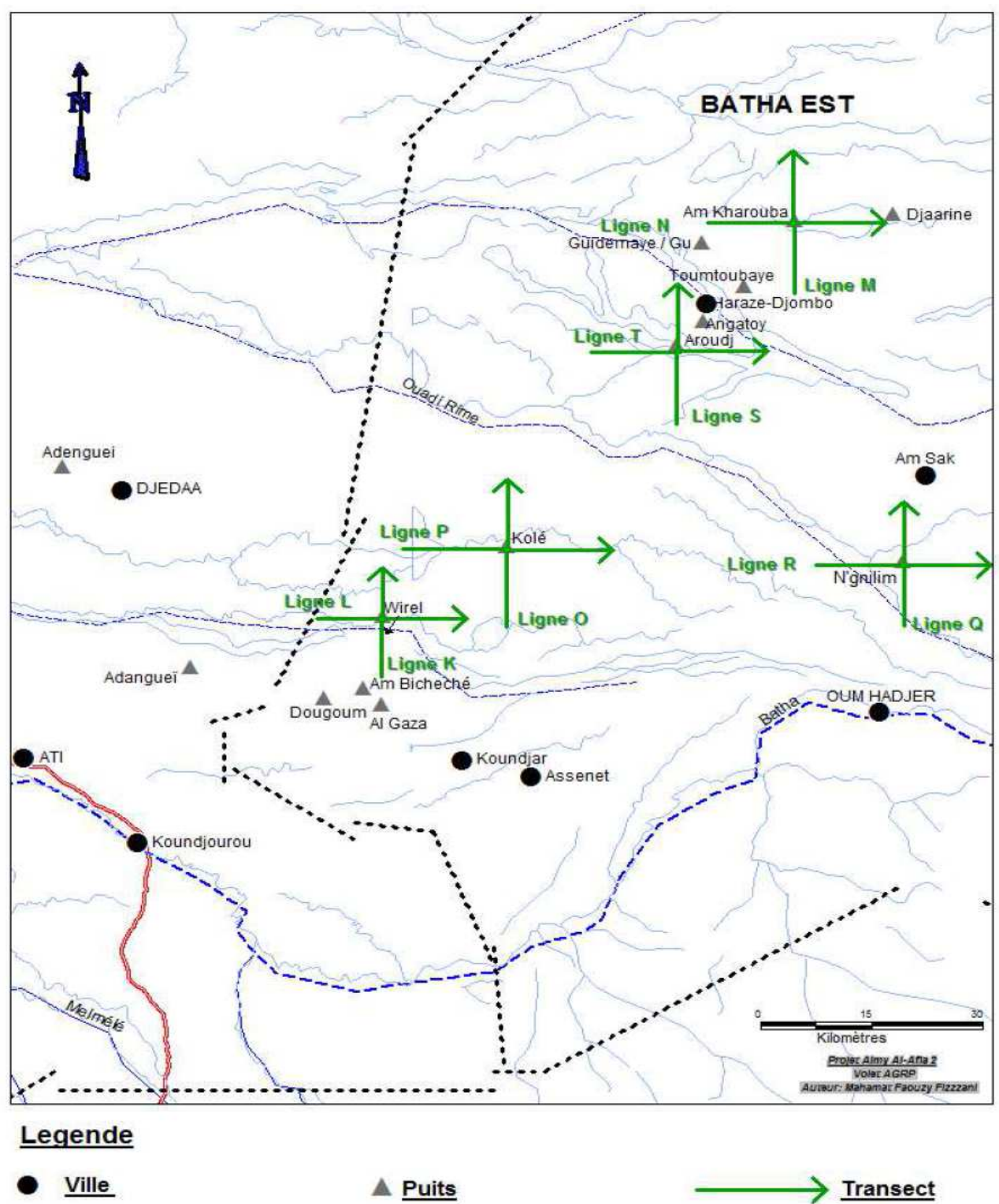

Figure 2 : Localisation des transects matérialisés dans les différents sites d'observation. 


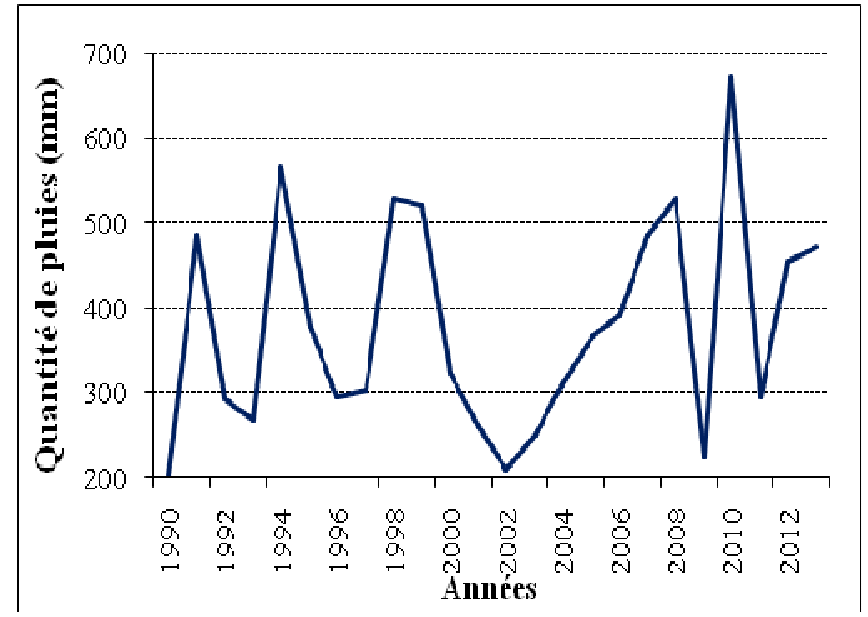

Figure 3 : Tendance évolutive de la pluviométrie annuelle dans la ville d'Oum Hadjer chef-lieu du Département de Batha Est.

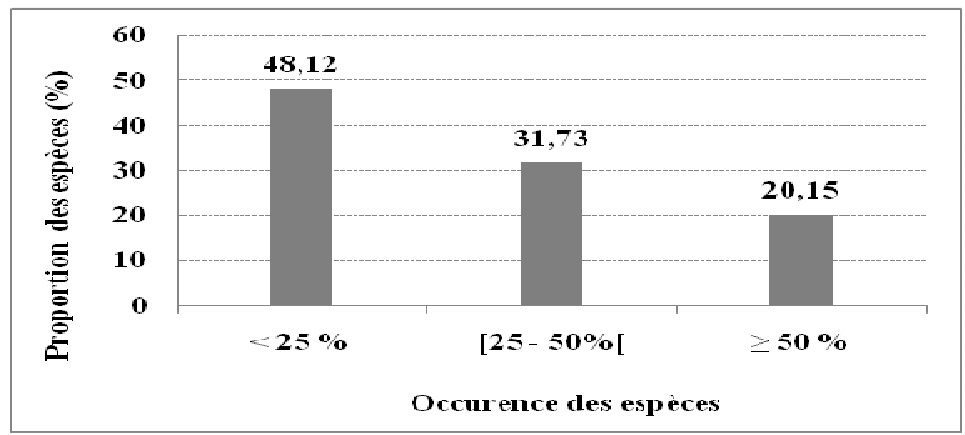

Figure 4 : Spectre des fréquences des espèces observées dans les différents sites.

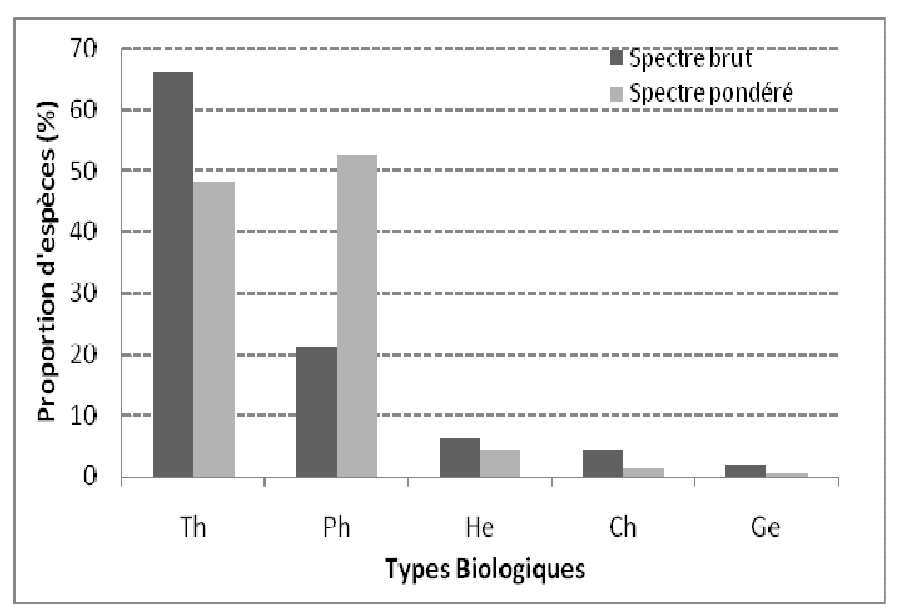

Figure 5 : Spectres brut et pondéré des types biologiques observés dans les différents sites. 
Tableau 1: Productivité et capacité de charge des pâturages des transects réalisés dans les différents sites.

\begin{tabular}{lcccc}
\hline $\begin{array}{l}\text { Unités de } \\
\text { pâturage }\end{array}$ & $\begin{array}{c}\text { Recouvrement } \\
\text { Ligneux }(\%)\end{array}$ & $\begin{array}{c}\text { Recouvrement } \\
\text { Herbacée } \mathbf{\% )}\end{array}$ & $\begin{array}{c}\text { Productivité } \\
(\mathbf{t} \text { MS/ha/an) }\end{array}$ & $\begin{array}{c}\text { Charge animale } \\
\text { (UBT / ha) }\end{array}$ \\
\hline U.P. I & 40 à 70 & 20 à 70 & 1 à 4 & 0,5 à 1 \\
U.P. II & 10 à 20 & 60 à 90 & 1 à 2 & 0,25 à 0,50 \\
U.P. III & 15 à 20 & 20 à 40 & 1 à 4 & 0,25 à 0,95 \\
U.P. IV & 10 à 40 & 40 à 60 & 1 à 3 & 0,25 à 0,70 \\
U.P. V & 20 à 30 & 40 à 70 & 1 à 3 & 0,25 à 0,60 \\
U.P. VI & 10 à 20 & 50 à 80 & 2 à 7 & 0,5 à 1,5 \\
\hline UP = Unité Pastoral & & &
\end{tabular}

Tableau 2: Richesse spécifiques, indices de diversité de Shannon et d'équitabilité de Pielou des différentes unités pastorales.

\begin{tabular}{lccc}
\hline $\begin{array}{l}\text { Unités de } \\
\text { végétation }\end{array}$ & $\begin{array}{c}\text { Richesses } \\
\text { spécifiques }\end{array}$ & $\begin{array}{c}\text { Indice de diversité de } \\
\text { Shannon (Bits) }\end{array}$ & Equitabilité de Pielou \\
\hline I & 35 & $2,96 \pm 0,55$ & $0,88 \pm 0,06$ \\
II & 48 & $2,52 \pm 0,55$ & $0,90 \pm 0,09$ \\
III & 43 & $2,73 \pm 0,32$ & $0,89 \pm 0,05$ \\
IV & 30 & $2,88 \pm 0,39$ & $0,89 \pm 0,07$ \\
V & 21 & $2,82 \pm 0,26$ & $0,90 \pm 0,05$ \\
VI & 29 & $2,84 \pm 0,38$ & $0,89 \pm 0,06$ \\
\hline
\end{tabular}

Tableau 3 : Comparaison des coefficients de similitude de Sorensen des différentes unités pastorales.

\begin{tabular}{lcccccc}
\hline Formations & I & II & III & IV & V & VI \\
\hline I & & & & & & \\
II & 0,22 & & & & & \\
III & 0,35 & 0,43 & & & & \\
IV & 0,23 & 0,38 & 0,31 & & & \\
V & 0,37 & 0,23 & 0,28 & 0,24 & & \\
VI & 0,21 & 0,31 & 0,24 & 0,25 & 0,28 & \\
\hline
\end{tabular}

\section{DISCUSSION}

L'analyse floristique et la fréquence des espèces ont montré que la flore des parcours pastoraux du Batha Est a été riche et variée. Cependant, toutes les familles recensées n'ont pas la même importance agrostologique. $\mathrm{La}$ prédominance des thérophytes est due au fait que le surpâturage n'affecte pas à lui seul directement la variabilité de la richesse en ce type biologique. Elle est beaucoup plus influencée par la pluviométrie (Westbrooke et al., 2005). La prééminence des thérophytes est d'une part, l'expression d'un climat sahélien à longue saison sèche et du substrat sableux et témoigne d'autre part, de leur adaptation aux stress climatiques (Tarhouni et al., 2007). Bien que faiblement représentées, les Hémicryptophytes indiquent une stabilité des sols et des conditions d'humidité relativement 
acceptables (Fournier et al., 2000). La forte proportion des poaceae et des légumineuses (Mimosaceae et Fabaceae) d'une part, et celle des Phanérophytes constitués en majorité d'espèces ligneuses fourragères (Maerua crassifolia, Acacia tortilis, Leptadenia pyrotechnica, Anogeissus leiocarpus et Balanites aegyptiaca) d'autre part, donne une certaine valeur aux différents parcours constituant un atout pour l'élevage dans la zone. Cela fait du Batha Est une bonne zone de repli de saison sèche. La faible proportion d'espèces fréquentes enregistrée $(20,15 \%)$ traduit l'homogénéisation de la végétation décrite par plusieurs auteurs (Jauffret et Lavorel, 2003 ; Tarhouni et al., 2007). Les graminées vivaces comme Andropogon gayanus, Sporobolus helvolus, Panicum maximum, Cymbopogon schoenantus et autres herbacées très recherchées par les éleveurs, telles que Blepharis linearifolia ont complètement disparu. Nos résultats corroborent ceux obtenus par Béchir et Mopaté (2015). Notre zone d'étude a aussi montré une succession d'unités pastorales constituées d'une végétation très claire à relativement dense. La très forte concentration des animaux et leur séjour plus ou moins prolongé a entrainé un développement des plages de Cenchrus biflorus, Dactyloctenuim aegyptium, Zornia glochidiata et Alysicarpus rigosus. En revanche, Aristida mutabilis, Chloris prieurii, Schoenefeldia gracilis et Tephrosia bracteolata ont colonisé les espaces peu fréquentés par les animaux. Cette situation est identique à celle décrite dans le Batha Ouest par Béchir et al. (2004) et Béchir et Mopaté (2015). La colonisation ou l'envahissement de la végétation par des espèces non appétées ou appétées occasionnellement comme Calotropis procera, Chrozophora senegalensis, Cassia obtusifolia, et Glinus latioides constitue également un signe d'une dynamique régressive. L'indice d'équitabilité de Pielou relativement élevé observé dans l'ensemble des unités pastorales indique un équilibre entre les espèces. Les unités II et V ont enregistré les plus grandes valeurs d'indice d'équitabilité, c'est-à-dire que leurs flores ont été réparties de façon plus homogène et équitable. Les richesses floristiques les plus importantes ont été observées dans les unités pastorales II et III, qui ont montré par contre les plus faibles indices de diversité. Ces résultats indiquent que la moyenne des espèces en nombre n'est pas synonyme d'un bon pâturage car les mauvaises espèces peuvent y être dominantes. Elle peut en revanche signifier une bonne diversité floristique. L'indice de diversité de Shannon (H) a été plus élevé pour les unités pastorales I et IV. En effet, ces unités font parties des formations les plus fréquentées en raison de leur proximité avec les points d'eau et aussi à cause de l'ombrage qu'offrent les forêts galeries aux animaux. L'indice de diversité de Shannon qui est plus élevé dans les parcours les plus pâturés corrobore les résultats obtenus par plusieurs auteurs (Fournier et al., 2000 ; Botoni-Liehoun et al., 2006 ; Akossoua et al., 2010) dans différentes zone agro-écologiques. Ceux-ci rapportent en effet, que le surpâturage d'un parcours entraine un maintien ou une augmentation de la diversité floristique. En revanche, Rakotoarimanana et al. (2008) précisent même que la diversité spécifique d'un herbage augmente quand la pression pastorale est moyenne et diminue lorsque la pression devient forte. En effet, le surpâturage provoque une nette domination de la végétation par une espèce ou par un petit nombre d'espèces (Hiernaux et Le Houerou, 2006). Les valeurs d'indices de similitude de Sorensen entre les différentes formations ont été inférieures à $50 \%$. Cela suppose que les six (6) unités pastorales aient été relativement différentes les unes des autres. En effet, la variation de l'indice de similarité entre les différentes unités pastorales identifiées semble être beaucoup plus influencée par la durée et l'intensité d'exploitation que par les conditions écologiques ou leur localisation géographique.

Comprises entre 1 à $7 \mathrm{tMS} / \mathrm{ha} / \mathrm{an}$ pour une charge annuelle théorique située entre 
0,25 et $1,5 \mathrm{UBT} / \mathrm{ha}$, les productivités des unités pastorales de la zone sont situées dans la limite établie par Toutain et al. (2000) pour la zone sahélienne. En ce qui concerne les effets des points d'abreuvement (puits ou mares) sur la végétation, leur aménagement pour valoriser les parcours du Batha Est a eu comme conséquence, l'afflux du cheptel autour de ces ouvrages. Au-delà des capacités de déplacement et de tolérance à la soif variables selon l'espèce animale, l'aire d'influence d'un point d'eau en saison sèche est circonscrite à un rayon d'environ 15 à 20 $\mathrm{km}$. Le nombre d'animaux pouvant être abreuvé ou la «charge animale » et le rythme d'exploitation des parcours environnant étant liés au débit d'un point d'eau. Dans notre zone d'étude, il a été noté une nette diminution de la richesse spécifique de la strate herbacée à proximité des points d'abreuvement, suite à l'exploitation intensive $\mathrm{du}$ milieu. Nos observations corroborent celles décrites dans la région par Béchir et Mopaté (2015). Ces auteurs affirment que la richesse floristique des parcours situés à proximité d'un point d'eau diminue. En effet, Cenchrus biflorus, Dactyloctenuim aegyptium, Zornia glochidiata et Alysicarpus rigosus ont dominé largement les proximités des ouvrages avec une nette tendance du premier à s'étendre de plus en plus loin. En raison de sa facile dissémination par les animaux, Cenchrus biflorus a semblé bien se maintenir en progressant et en s'étendant sur de vastes surfaces pour coloniser toutes les zones d'influence des points d'abreuvement. Près des abreuvoirs, sur quelques dizaines ou centaines de mètres, le sol a été complètement nu ou occupé par d'espèces non ou très peu appétées comme Cassia obtusifolia et Trianthema portulacastrum. Aux alentours immédiats des ouvrages, une diminution de la porosité des sols a été aussi observée notamment en profondeur. Entre trois (3) et quinze (15) km, l'effet de la pâture s'est traduit par l'abondance de Cenchrus biflorus associée à Dactyloctenuim aegyptium. Ces espèces ont bénéficié de l'importation d'azote et des effets défavorables sur les espèces plus sensibles au piétinement. Elles ont pu ainsi coloniser de grandes surfaces à la faveur d'une bonne fertilisation du sol. Dans les zones les plus éloignées des puits (plus de 15 $\mathrm{km}$ ), les effets de la pression animale ont varié selon le type de sol. Les associations Schoenefeldia gracilis, Aristida mutabilis et Eragrostis tremula y ont été les plus dominantes. La densité des ligneux a aussi diminuée à proximité des point d'abreuvement. En effet, leur recouvrement a été plus faible à l'approche d'un ouvrage. De plus, une modification de la physionomie de la végétation ligneuse est observée sur un rayon de 1 à $8 \mathrm{~km}$ autour des points d'abreuvement surtout ceux implantés sur sols sableux. Nos résultats ont été semblables à ceux de Diop et al. (2005) qui ont observé une diminution importante de la densité ligneuse autour des forages sur un même rayon et sur les mêmes types de sols. En effet, la très forte concentration animale autour des points d'abreuvement en saison sèche a beaucoup pesé sur les peuplements des Maerua crassifolia, Boscia senegalensis, Balanites aegyptiaca et surtout sur les jeunes pousses. Ces essences ont montré une forme rabougrie avec une croissance beaucoup plus en épaisseur à cause de la consommation systématique des bourgeons foliaires axillaires et terminaux. Cependant, le stade final avec la substitution de Calotropis procera tel que décrit par Béchir et Mopaté (2015) dans le Batha Ouest n'a pas été observé dans la zone.

Concernant la biomasse herbacée, aucun effet lié à la présence d'un point d'abreuvement n'a été observé. La production primaire a été parfois beaucoup plus élevée à proximité des points d'eau que dans les pâturages les plus éloignés. Cela s'explique par la forte concentration des animaux autour des points d'eau aux heures de pointe, entraînant une exploitation pastorale importante. En effet, la diversité floristique des herbacées des zones les plus pâturées augmente (Rakotoarimanana et Grouzi, 2006). La productivité des herbages n'est pas 
affectée par le voisinage d'un point d'eau. Toutefois, nous convenons avec Rakotoarimanana et al. (2008) qui affirment que le pâturage augmente le recouvrement des herbages mais diminue la biomasse et la phytomasse herbacées.

L'encroûtement superficiel des sols observé dans les zones sableuses de notre zone d'étude n'est pas attribuable aux seuls effets de la pâture et du piétinement. Ce phénomène a été également observé dans des zones parfois très éloignées d'un point d'eau. Ils sont plutôt attribuables à des facteurs naturels comme la granulométrie (Diop et al., 2005). En effet, selon ces auteurs, l'apparition des croûtes de battance ou tâches nues pelliculaires sont très rares sur les sols à textures plus grossières car ceux-ci sont moins favorables à leur formation.

\section{Conclusion}

L'étude a permis de connaître la diversité floristique et les potentialités fourragères et productives des parcours pastoraux du Batha Est. Elle constitue une base indispensable pour mieux orienter les interventions en matière de gestion de l'espace pastoral dans la zone. L'inventaire floristique a montré une flore riche à importance agrostologique très variable. Chacune de six (6) unités pastorales identifiées et décrites est caractérisée par une structure et une diversité floristique fortement influencées par les conditions édaphiques, climatiques et anthropiques locales. Bien que constitués d'espèces végétales nombreuses et variées et à grande majorité appétées, ces différents parcours ont été d'une valeur pastorale assez médiocre. Suite à leur exploitation intensive, la richesse spécifique de la strate herbacée a considérablement diminuée. L'étude a montré un dépassement de la charge réelle des parcours. Cela donne lieu à un surpâturage qui résulte du maintien trop prolongé des troupeaux sur les aires de pâture. Il faudra résoudre le problème de la faible capacité des parcours à supporter la charge du bétail qui les fréquente. En effet, la mauvaise répartition du cheptel combinée à la diminution progressive de la complémentarité entre les espaces et les communautés et la présence de gros troupeaux ont engendré une rupture de l'équilibre entre la ressource disponible et les besoins du cheptel. Il a été également observé une forte colonisation agricole de l'ensemble de l'espace pastoral, dans un mouvement massif d'appropriation. Cette dynamique vient concurrencer l'élevage pastoral et rend très difficile et conflictuel l'accès aux ressources et la mobilité. Ainsi, en raison de la gratuité de leur accès qui a considérablement changé leurs modes d'utilisation en saison sèche, la gestion des parcours pastoraux à proximité des points d'abreuvement semble être affectée de façon durable mais non irréversible. Dans ces conditions de libre accès aux points d'eau, une exploitation rationnelle de l'espace pastoral paraît difficile mais pas impossible. Aussi, toute stratégie de sécurisation de la mobilité et de gestion durable des ressources doit-elle s'inscrire dans une dynamique d'aménagement du territoire et de gestion concertée de l'espace et des ressources.

\section{REMERCIEMENTS}

Les auteurs remercient le Programme d'Hydraulique Pastoral au Tchad Central (Almy Al Afia Phase II) pour le financement des activités de recherches ayant abouti à cette publication ainsi que toute l'équipe du volet AGRP pour sa participation dans la collecte des données.

\section{REFERENCES}

Béchir AB, Mopaté LY. 2015. Analyse de la dynamique des pâturages autour des ouvrages hydrauliques des zones pastorales du Batha Ouest au Tchad. Afrique Science, 11(1): 212-226.

Béchir AB, Wiese M, Sabra D, Koundja N. 2004. Ecologie en milieu pastoral: cas de la zone nord du Programme d'hydraulique pastorale au Tchad Central (Batha-ouest), LRVZ, 32 p. 
Bonnet B, Marty A, Demante M. 2005. Hydraulique et sécurisation des systèmes pastoraux au Sahel: appui à la gestion locale. Demarches \& méthodes, IRAM, p. 28.

Botoni-Liehoun E, Daget P, César J. 2006. Activités de pâturage, biodiversité et végétation pastorale dans la zone Ouest du Burkina Faso. Revue Élev. Méd. Vét. Pays Trop., 59(1-4): 31-38.

Braun-Blanquet J. 1932. Plant Sociology. The Study of Plant Communities (English Translation of "pflazensoziologieFuller», GD, Conard HS (eds). Université de Chicago: Chicago, USA.

Diop AT, Sy O, Ickowicz A, Touré I. 2005. Politique d'hydraulique et gestion de l'espace et des ressources dans la région sylvopastorale du Sénégal (Ferlo). Synthèse des études sur la politique hydraulique en Zone Sylvopastorale (ZSP) ou Ferlo, 9 p.

Fournier A, Yoni M, Zombré P. 2000. Les jachères à Andropogon gayanus en savane soudanienne dans l'ouest du Burkina Faso: flore, structure, déterminants et fonction dans l'écosystème. Etude Flore Végétation. Burkina Faso, 5: 3-32.

Hiernaux P, Le Houerou HN, 2006. Les parcours du Sahel. Sécheresse, 17(1-2): 51-71.

Jauffret S, Lavorel S. 2003. Are plant functional types relevant to describe degradation in arid, southern Tunisian steppes. J. Veg. Sci., 14: 399-408.

Kouassi AF, Adou CY, Ipou IJ, Kamanzi K. 2010. Diversité floristique des zones côtières pâturées de la Côte d'Ivoire : cas $\mathrm{du}$ cordon littoral Port-Bouët-Grand-
Bassam (Abidjan). Sciences \& Nature, 7(1): 69-86.

Rakotoarimanana V, Gondard H, Ranaivoarivelo N, Carriere S. 2008. Influence des pâturages sur la diversité floristique, la production et la qualité fourragère d'une savane des Hautes Terres malgaches (Région de Fianarantsoa). Sécheresse, 19(1): 39-46.

Shannon CE. 1948. A mathematical theory of communication. Bell System Technical Journal, 27: 379-423.

Sorensen CE. 1948. A method of establishing groups of equal amplitude in plant sociology based on similarity of species content. K. Danske Vidensk. Selsk, 5: 134.

Tarhouni M, Ben Salem F, Ouled Belgacem A, Henchi B, Neffati M. 2007. Variation de la richesse floristique en fonction du gradient de pâturage au voisinage de points d'eau. Sécheresse, 18(4): 9-234.

Thébaud B. 1990. Politique d'hydraulique pastorale et gestion de l'espace au Sahel. Cah. Sci. Hum., 26(1-2) ; 13-31.

Toutain B, Touré O, Réounodji F. 2000. Etude prospective de la stratégie nationale de gestion des ressources pastorales au Tchad. Rapport final, $102 \mathrm{p}$.

Toutain B, Marty A, Bourgeot A, Ickowicz A, Lhoste P. 2012. Pastoralisme en zone sèche. Le cas de 1'Afrique subsaharienne. Les dossiers thématiques du CSFD. $\mathrm{N}^{\circ}$, février 2012. CSFD/Agropolis International, Montpellier, France, $60 \mathrm{p}$.

Westbrooke ME, Florentine SK, Milberg P. 2005. Arid land vegetation dynamic after a rare flooding event: influence of fier and grazing. J. Environ Manage, 61: 60249. 